\title{
Anomalous phenomenon in hydrogen gas sensors based upon pyridylimidazoperylene or pyridylimidazonaphthalene
}

\author{
Keita Hirao, ${ }^{1}$ Yuichiro Tsukada, ${ }^{1}$ Shigeru Suzuki, ${ }^{1}$ Hiroo Takahashi, ${ }^{1}$ and Jin Mizuguchi ${ }^{1}$ a) \\ ${ }^{1}$ Department of Applied Physics, Graduate School of Engineering, Yokohama National University, \\ Yokohama, Kanagawa 240-8501, Japan
}

(Received 13 August 2007; accepted 3 January 2008; published online 11 March 2008)

\begin{abstract}
We have been involved in the research and development on $\mathrm{H}_{2}$ gas sensors utilizing a high proton affinity of the pyridyl rings integrated in organic pigments such as diketopyrrolopyrroles, peryleneimides, and copperphthalocyanes. Our sensors exhibit a remarkable increase in electrical conductivity by three orders of magnitude even under $0.01 \% \mathrm{H}_{2}$. However, an anomalous phenomenon has been observed in the title compounds (pyridylimidazoperylene and pyridylimidazonaphthalene) that shows an additional increase in conductivity (i.e., reverse of the sensor current) as soon as $\mathrm{H}_{2}$ shuts off. The unusual effect has been investigated in the present investigation on the basis of the crystal structure, molecular orbital calculations, and UV-visible/IR spectra with special attention to the sign of the charge carriers before and after exposure to $\mathrm{H}_{2}$. As a result, the pyridylimidazole ring is found to be responsible for the unusual effect that involves two protonation sites. In addition, the charge carriers have been determined to be solely electrons in the build up of the sensor current; whereas both electrons and protons are involved in the decay process. The contribution of the proton conduction in the decay process prevails over the electron current, ending up with the reverse in sensor current. (C) 2008 American Institute of Physics.
\end{abstract}

[DOI: $10.1063 / 1.2890425$ ]

\section{INTRODUCTION}

The title compounds pyridylimidazoperylene (PIP) [Fig. 1(a)] and pyridylimidazonaphthalene (PIN) [Fig. 1(b)] are pyridylderivatives of benzimidazoperylene and benzimidazonaphthalene that are industrially important pigments. ${ }^{1}$

With the advent of fuel cells based upon $\mathrm{H}_{2}, \mathrm{H}_{2}$ gas sensors have attracted attention, because hydrogen is the smallest atom and, thus, can leak easily. In addition, it can catch fire, for example, from a spark. ${ }^{2}$ We have been so far involved in the research and development of a $\mathrm{H}_{2}$ gas sensor utilizing a high proton affinity of dipyridyldiketopyrrolopyrrole (DPPP) [Fig. 2(a) $]^{3}$ and extended our system to larger chromophores such as peryleneimides ([ortho-pyridyl perylene (OPP), meta-pyridyl perylene (MPP), and parapyridyl perylene (PPP)] [Fig. 2(b) $]^{4}$ and copperphthalocyanines $(\mathrm{CuPPc})[\text { Fig. 2(c) }]^{5}$ In these sensors, proton capture at the $\mathrm{N}$ atom of the pyridyl ring induces a remarkable reduction in resistivity by three orders of magnitude even under $0.01 \% \mathrm{H}_{2}$. In addition, the build up and build down of the sensor signal are quite normal, as shown in Fig. 3(a), where the response curve of a MPP-based sensor is displayed. However, in PIP, we encountered an anomalous phenomenon [Fig. 3(b)] that shows an additional increase in sensor signal even after the shutoff of $\mathrm{H}_{2}$ and the signal becomes maximum in $200 \mathrm{~s}$ and then comes back to the initial state in $300 \mathrm{~s}$. The extent of the unusual effect depends greatly on the $\mathrm{H}_{2}$ exposure time. The longer the exposure time is, the larger and the longer is the abnormal effect, indicating that the effect is closely associated with reactions of $\mathrm{H}_{2}$ on the proton accepting sites. Furthermore, we have clarified that

${ }^{a)}$ Electronic mail: mizu-j@ynu.ac.jp the present effect is a characteristic of those compounds which include pyridylimidazole rings such as PIP or PIN.

The purpose of the present investigation is to clarify the anomalous effect in PIP or PIN on the basis of the crystal structure, molecular orbital calculations, and UV-visible/IR spectra with special attention to the sign of the charge carriers before and after exposure to $\mathrm{H}_{2}$, as measured by the Seebeck method. Since PIN behaves exactly the same way as PIP, the present paper deals mostly with the results of PIP.

\section{EXPERIMENT}

\section{A. Synthesis and crystal growth}

PIP was synthesized by heating perylene-3,4,9,10tetracarboxylic dianhydride and diaminopyridine at $490 \mathrm{~K}$ in

(a)

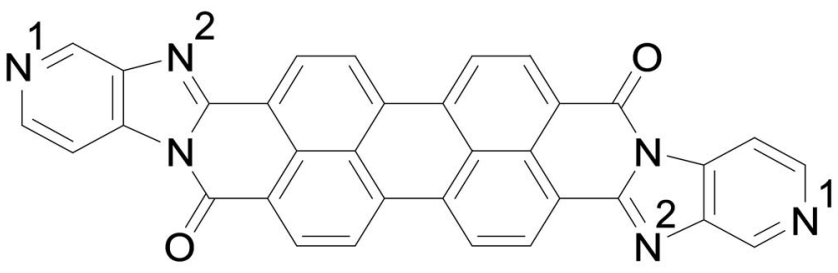

(b)

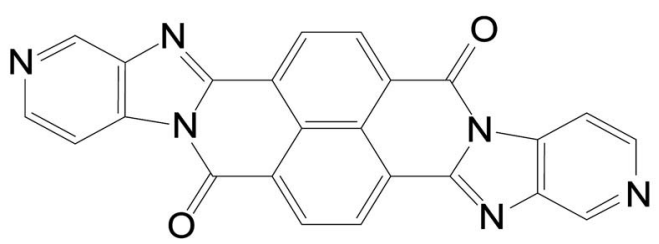

FIG. 1. Molecular conformation: (a) PIP and (b) PIN. N1 and N2 are the first and second protonation sites used for later discussion. 
(a)<smiles>O=C1NC(c2ccncc2)=C2C(=O)NC(c3ccncc3)=C12</smiles>

(b)<smiles></smiles>

$\mathbf{R}:$<smiles>Cc1ccc(-c2cccnc2)cn1</smiles>

(c)

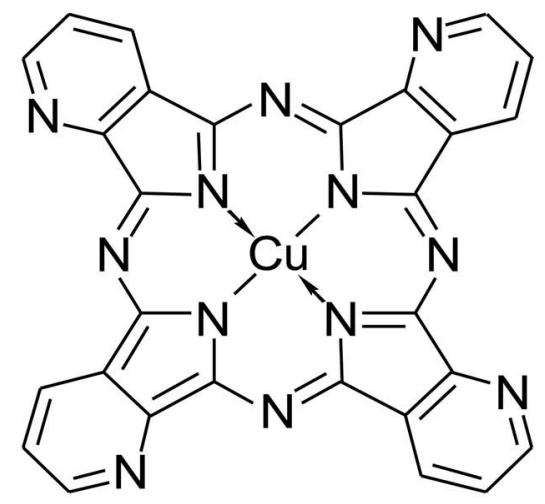

FIG. 2. (a) DPPP, (b) Peryleneimides (OPP, MPP, and PPP), and (c) coppertetra-2,3-pyridinoporphyradine ( $\mathrm{CuPPc})$.

phenol for $5 \mathrm{~h}$. Likewise, PIN was prepared using naphthalene-3,4,9,10-tetracarboxylic dianhydride. The products were then purified by sublimation at $490 \mathrm{~K}$, using a two-zone furnace. ${ }^{6}$ Single crystals of PIP or PIN were grown from the vapor phase in a closed system.

\section{B. Fabrication of $\mathrm{H}_{2}$ sensors and the operation principle}

Figure 4 shows the interdigital electrode made of indium tin oxide (ITO) (transparent) prepared by photolithographic (a)

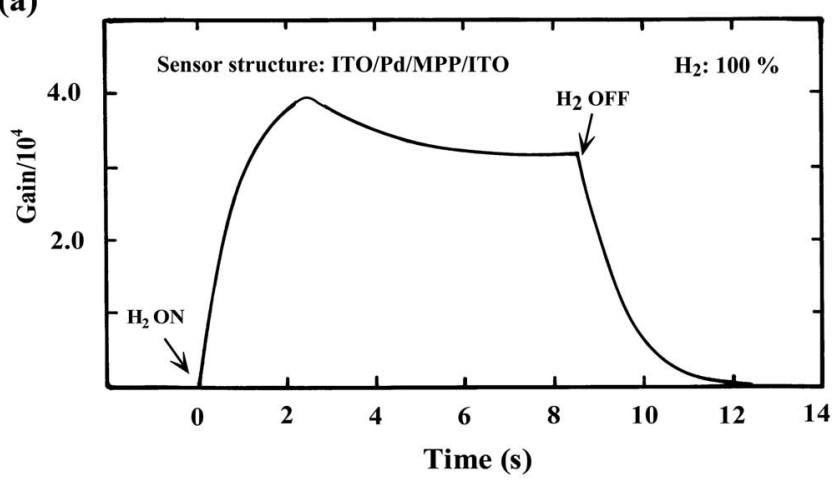

(b)

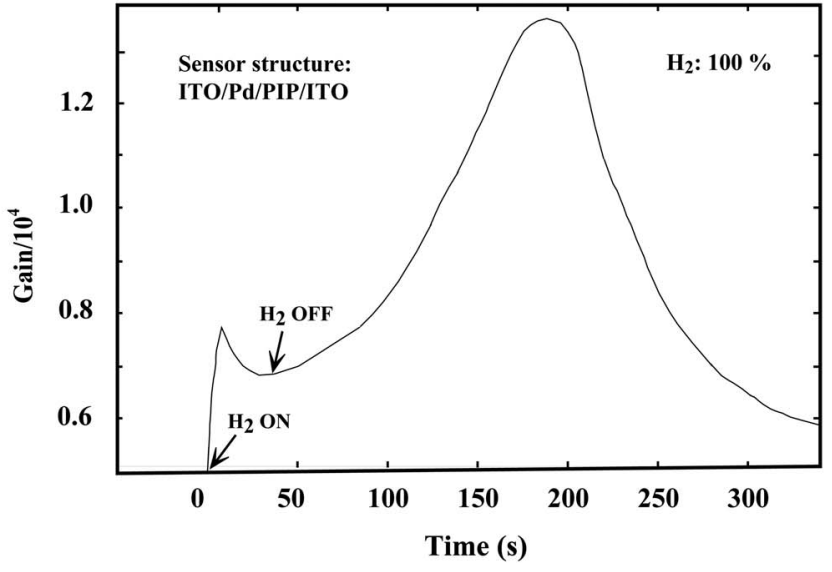

FIG. 3. (a) Response curve of the $\mathrm{H}_{2}$ sensor based upon MPP and (b) response curve of the $\mathrm{H}_{2}$ sensor based upon PIP, showing the anomalous characteristics.

technique. Then, Pd was sputtered directly onto the electrode by a sputtering apparatus (E-1030 Ion Sputter from Hitachi Corporation) in such a way as to form islands of $\mathrm{Pd}$ so as to avoid Pd-Pd contacts [Fig. 4(b)] (thickness: about $3 \AA$ ). After that, a thin layer of PIP or PIN was applied by vacuum evaporation (Tokyo Vacuum Co., Ltd.: model EG240) to the thickness of about $300 \AA$. The sensor is slightly colored but still transparent. It is also important to note that the presence of a minute amount of $\mathrm{Pd}$ is crucial for the dissociation of $\mathrm{H}_{2}$ into $\mathrm{H}^{+}$.

(a)

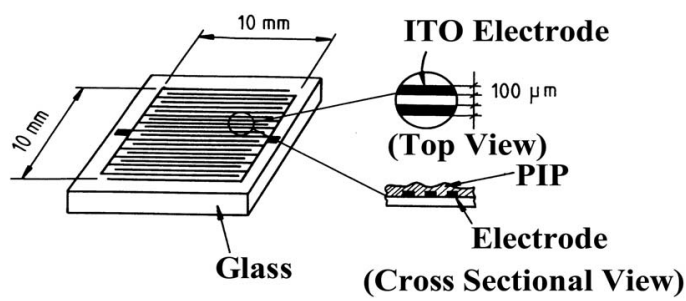

(b)

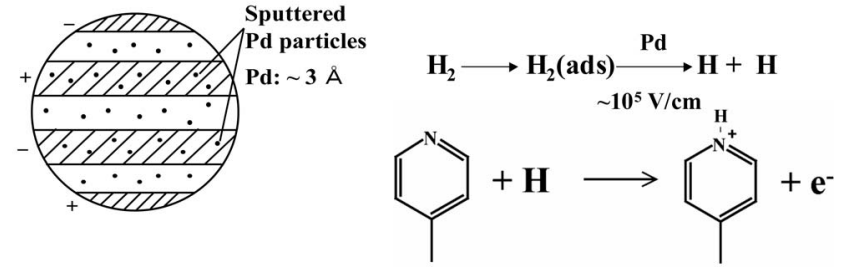

FIG. 4. (a) Interdigital electrodes and (b) magnified Pd-sputtered electrodes. 


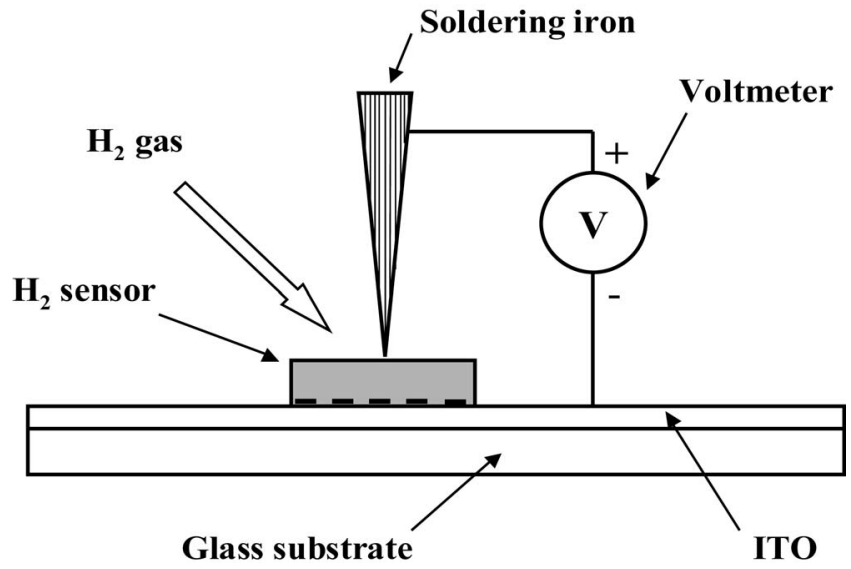

FIG. 5. Experimental setup for measurements of the Seebeck coefficient.

The sensing process proceeds in the following way. $\mathrm{H}_{2}$ is first adsorbed on the surface of, for example, DPPP, and diffuses into the bulk where $\mathrm{H}_{2}$ encounters sputtered Pd. Then, $\mathrm{H}_{2}$ dissociates into hydrogen atoms under a high electric field: $\mathrm{H}_{2} \rightarrow \mathrm{H}+\mathrm{H}$. At this moment, the $\mathrm{N}$ atom of the pyridyl ring of DPPP (strong proton acceptor) captures the proton by releasing an electron that contributes to the sensor current. The sensor exhibits a remarkable reduction of resistivity by about three orders of magnitude even under $0.01 \%$ $\mathrm{H}_{2}$. The decay process when $\mathrm{H}_{2}$ is shut off is supposed to proceed with release of $\mathrm{H}^{+}$from $\mathrm{N}-\mathrm{H}^{+}$to recombine with a free electron: $\mathrm{H}^{+}+e^{-} \rightarrow 1 / 2 \mathrm{H}_{2}$ (desorption).

\section{Experimental setup for measurements of the Seebeck effect}

Figure 5 shows the experimental setup for measurements of the Seebeck effect for the determination of the charge carrier. ${ }^{7}$ This method measures thermoelectric power that appears between hot and cold ends of a material. The hot end is made by a soldering iron with a small spring on top maintained at $100{ }^{\circ} \mathrm{C}$; whereas the cold end is the ITO electrode at room temperature. If the potential of the hot end is positive, then the carriers are electrons. Similarly, if the potential is negative, the charge carriers are holes or cations. Since the measurement of the Seebeck effect requires a temperature gradient along the direction of the film thickness, the PIP or PIN layer in the $\mathrm{H}_{2}$ gas sensor was prepared thicker (about $1700 \AA$ ) than the standard one (about $300 \AA$ ).

\section{UV-visible and IR spectra}

UV-visible spectra of PIP- or PIN-based $\mathrm{H}_{2}$ sensors were recorded on a UV-2400PC spectrophotometer (Shimadzu) before and after $\mathrm{H}_{2}$. Similarly, IR spectra of evaporated PIP or PIN were measured on a microsampling Fourier transform IR spectrometer from JASCO (model MFT-2000) before and after $\mathrm{H}_{2}$. The sample for IR measurements were prepared by vacuum evaporation directly onto a $\mathrm{KBr}$ disk with a trace of Pd (PIP or PIN thickness: about $2000 \AA$ ).
TABLE I. Crystallographic parameters for PIP and PIN.

\begin{tabular}{lcc}
\hline \hline & $\mathrm{PIP}$ & $\mathrm{PIN}$ \\
\hline Formula & $\mathrm{C}_{34} \mathrm{H}_{14} \mathrm{~N}_{6} \mathrm{O}_{2}$ & $\mathrm{C}_{24} \mathrm{H}_{10} \mathrm{~N}_{6} \mathrm{O}_{2}$ \\
Crystal System & Triclinic & Monoclinic \\
Space group & $P 1$ & $P 2_{1} / n$ \\
Molecular symmetry & $C_{i}$ & $C_{i}$ \\
$Z$ & 1 & 2 \\
$a(\AA)$ & $6.578(3)$ & $9.6635(2)$ \\
$b(\AA)$ & $9.123(5)$ & $8.7803(2)$ \\
$c(\AA)$ & $9.555(5)$ & $10.7870(3)$ \\
$\alpha(\mathrm{deg})$ & $103.05(3)$ & 90 \\
$\beta(\mathrm{deg})$ & $73.73(3)$ & $111.45(13)$ \\
$\gamma(\mathrm{deg})$ & $90.48(3)$ & 90 \\
$V\left(\AA^{3}\right)$ & $557.2(5)$ & $851.87(4)$ \\
$d\left(\mathrm{~g} / \mathrm{cm}^{3}\right)$ & 1.605 & 1.615 \\
\hline \hline
\end{tabular}

\section{E. Molecular orbital (MO) calculations}

Geometry optimization has been carried out on the trans form of PIP by means of the AM1 Hamiltonian of MOPAC93. ${ }^{8}$ The spectroscopic calculations were then performed on the optimized geometry using the INDO/S Hamiltonian in ZINDO program package. ${ }^{8}$

\section{RESULTS AND DISCUSSION}

\section{A. Crystal structure of PIP and PIN}

Both PIP and PIN are found to be of the trans form according to the structure analysis. However, refinement of both structures is not entirely completed yet because of the poor quality of the single crystals used. Only the crystallographic parameters are presented for PIP and PIN in Table I.

\section{B. Electron density of the $\mathbf{N}$ atoms and absorption bands}

Table II shows the electron density of the N1 and N2 atoms (see Fig. 1 for the designation) in the initial state, as well as in the first and second protonation states. The absorption bands are also presented together with their oscillator strength for the corresponding states. It is important to note that the electron density is highest at N1, followed by $\mathrm{N} 2$ and that both $\mathrm{N}$ sites are capable of accepting protons. The presence of two protonation sites is a characteristic of the pyridylimidazole ring integrated in PIP or PIN and these are not found in DPPP, ${ }^{3}$ peryleneimides (OPP, MPP, and PPP), ${ }^{4}$ and CuPPc. $^{5}$ As judged from the electron density at the $\mathrm{N}$ atoms, the first protonation is supposed to occur at N1 (outer N atom) and then at N2 (inner N atom). In parallel, the heat of formation increases with the first and second protonations, as shown in Table II. The absorption band is then shifted toward shorter wavelengths upon first protonation while toward longer wavelengths upon second protonation.

\section{Absorption spectra of PIP-based $\mathbf{H}_{\mathbf{2}}$ gas sensors}

Figure 6 shows the absorption spectrum of the transparent $\mathrm{H}_{2}$ sensor based upon PIP as prepared, and those before and after exposure to $\mathrm{H}_{2}$. The PIP-based sensor as prepared exhibits blue but the color changes gradually to violet as 
TABLE II. Electron density and optical absorption bands as deduced from MO calculations.

Electron density
$\begin{gathered}\text { Heat of formation } \\ (\mathrm{kcal} / \mathrm{mol})\end{gathered}$

soon as the sensor is exposed to air ("before" state). Then, the color becomes sky blue upon exposure to $\mathrm{H}_{2}$ ("after" state). Finally, the color comes back to violet again (before state). The absorption maximum of the spectra is first hypsochromically and then bathochromically displaced on going from the as prepared state, via before state to the after state. This tendency is in good agreement with the results of MO calculations carried out on the initial state as well as on the first and second protonated states.

Furthermore, the color change from blue (as prepared state) to violet (before state) is remarkably accelerated when exposed to water vapor, indicating that water moisture in air is involved in the present color change. Another important fact is that the color change from blue to violet occurs as well due to water vapor in evaporated PIP alone in the absence of Pd. However, no color change from violet to sky blue due to $\mathrm{H}_{2}$ takes place. This occurs only in the $\mathrm{H}_{2}$ sensors which include $\mathrm{Pd}$. The present result indicates that only protons $\left(\mathrm{H}^{+}\right)$can induce the color change and not $\mathrm{H}_{2}$. On the basis of the above experimental results, we assume that the color change from blue (as prepared state) to violet is due to protonation at $\mathrm{N} 1$ due to water moisture in air since N1 is more reactive than $\mathrm{N} 2$ as judged from the electron density.

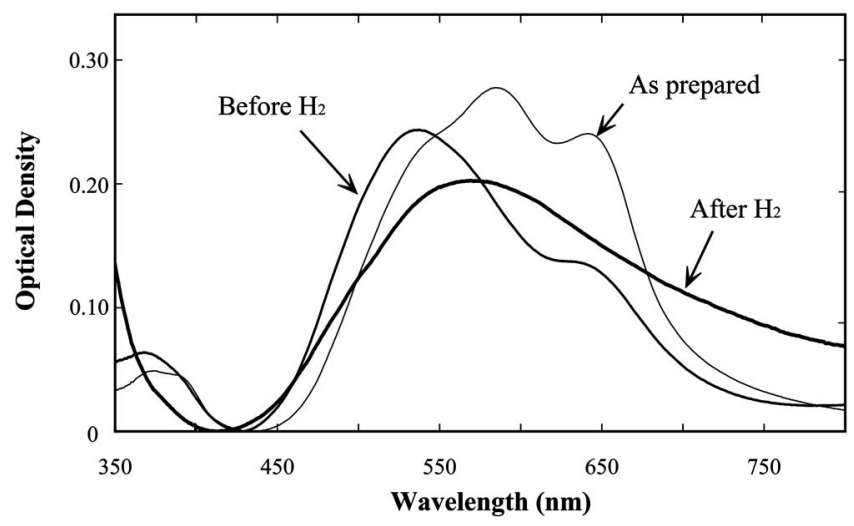

FIG. 6. Absorption spectra of the $\mathrm{H}_{2}$ sensors based on PIP: as prepared, before introduction of $\mathrm{H}_{2}$, and after shut off of $\mathrm{H}_{2}$. Thickness of evaporated PIP: about $300 \AA$.
Furthermore, the before state (violet) to the after state due to $\mathrm{H}_{2}$ involves a protonation at the $\mathrm{N} 2$ site in terms of $\mathrm{H}^{+}$dissociated from $\mathrm{H}_{2}$ in the presence of $\mathrm{Pd}$.

\section{Measurement of the IR spectra}

Figure 7(a) shows the IR spectra of the $\mathrm{H}_{2}$ sensor measured for state 1 (as prepared), state 2 (exposure to air), state $3\left(\mathrm{H}_{2} \mathrm{on}\right)$, and state $4\left(\mathrm{H}_{2}\right.$ off $)$ together with the standard IR spectra for (hydrogen-bonded) water [Fig. 7(b) $]^{9}$ and water moisture [Fig. 7(c)]. ${ }^{9}$ State 1 corresponds to the state as prepared (blue), state 2 (exposure to air: violet), state $3\left(\mathrm{H}_{2}\right.$ on: sky blue), and state 4 ( $\mathrm{H}_{2}$ off: violet). The broadband around $3400 \mathrm{~cm}^{-1}$ in states 2 and 4 is indicative of the hydrogenbonded $\mathrm{OH}$ or NH stretching [see Fig. 7(b)]. The noisy bands in the region between 3400 and $3900 \mathrm{~cm}^{-1}$ in state 3 are assigned to those of water moisture in the absence of hydrogen bonds [see Fig. 7(c)]. It is also important to remember that the noisy IR spectra due to isolated water is not observed in our $\mathrm{H}_{2}$ sensors based upon DPPP, ${ }^{3}$ peryleneimides (OPP, MPP, and PPP), ${ }^{4}$ and CuPPc. ${ }^{5}$ This indicates that the anomalous effect is associated with the appearance of the noisy IR spectra of isolated water.

\section{E. Carrier determination based upon the Seebeck effect}

Figure 8 shows the Seebeck potential of PIP-based $\mathrm{H}_{2}$ sensors when $\mathrm{H}_{2}$ is on and off. Since the positive sign appears upon introduction of $\mathrm{H}_{2}$, the charge carriers are determined to be electrons. As soon as the shutoff of $\mathrm{H}_{2}$, the positive potential decays quickly, indicating that the number of electrons is reduced. However, at this moment, the sensor current starts increasing, as shown in Fig. 3(b). This clearly indicates that the holes or cations are produced after shutoff of $\mathrm{H}_{2}$ that contribute to the sensor current.

\section{F. Proposed mechanism for the anomalous effect}

On the basis of the observed results, we propose our mechanism for the anomalous effect, as summarized in Fig. 9. In process (a) where the color change takes place from the 
(a)

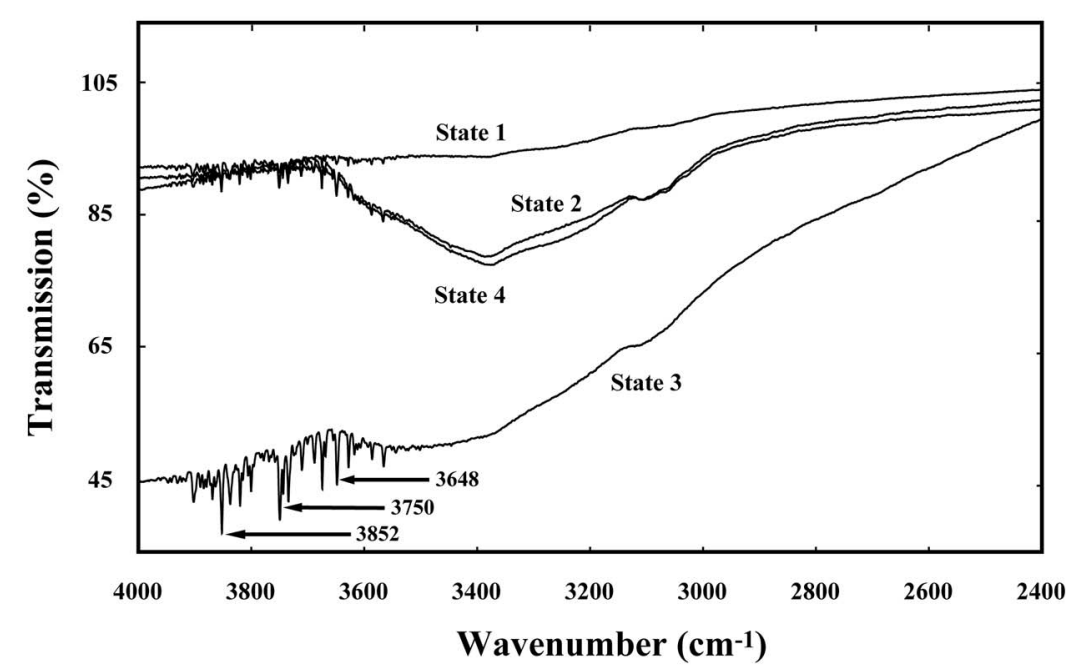

(b)

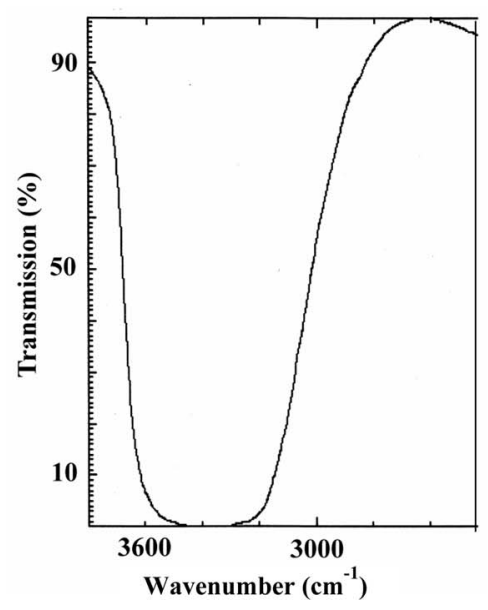

(c)

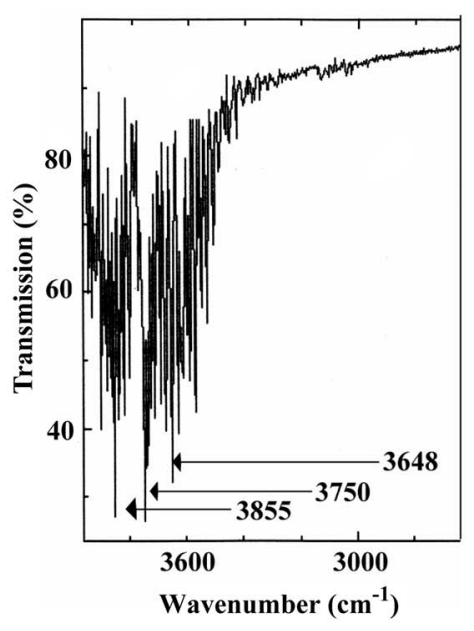

FIG. 7. (a) IR spectra of the $\mathrm{H}_{2}$ sensor: state 1 (as prepared), state 2 (exposure to air), state $3\left(\mathrm{H}_{2}\right.$ on), and state 4 ( $\mathrm{H}_{2}$ off), (b) IR spectrum for (hydrogen-bonded) water (Ref. 8) and IR spectra of water moisture in the molecular state (Ref. 8). as prepared (blue) to the before state (violet), water moisture in air is involved and is supposed to react with $\mathrm{N} 1$ while the $\mathrm{N} 2$ remains intact: $\mathrm{N} 1$ (pyridyl rings) $+\mathrm{H}_{2} \mathrm{O} \rightarrow \mathrm{N}^{+} \mathrm{H} \cdots \mathrm{OH}^{-}$, where the $\mathrm{OH}^{-}$is hydrogen-bonded to $\mathrm{NH}^{+}$as a counter ion. This gives the $\mathrm{OH}^{-}$stretching band around $3400 \mathrm{~cm}^{-1}$ [Fig. 7(a)]. The color change due to the protonation at the N1 site is in good agreement with the result of MO calculations (Table II).

In process (b), when $\mathrm{H}_{2}$ is introduced (violet $\rightarrow$ sky blue), the proton arising from the dissociation of $\mathrm{H}_{2}$ in the presence of $\mathrm{Pd}$ attacks the $\mathrm{OH}^{-}$at the $\mathrm{N} 1$ site to give isolated water [IR spectra in state 3 in Fig. 7(a)] and also to release one electron. At the same time, protonation occurs at N2 to yield one electron. The latter is the process which occurs usually in DPPP, ${ }^{3}$ peryleneimides (OPP, MPP, and PPP), ${ }^{4}$ and $\mathrm{CuPP}^{5}$ Both electrons released from the reactions at $\mathrm{N} 1$ and $\mathrm{N} 2$ contribute to the sensor current. The electron conduction is experimentally borne out by the Seebeck measurements (Fig. 8).

In process (c), when $\mathrm{H}_{2}$ is shut off (sky blue $\rightarrow$ violet), the conventional decay process ${ }^{3-5}$ takes place at the N2 site as observed in our DPPP, ${ }^{3}$ peryleneimides (OPP, MPP, and PPP), ${ }^{4}$ and CuPPc. ${ }^{5}$ This is the process to release a $\mathrm{H}^{+}$from
$\mathrm{N}-\mathrm{H}^{+}$and to acquire a free electron to recombine to give $1 / 2 \mathrm{H}_{2}$ (desorption) (see Sec. III B). This process is known to proceed quite fast in several seconds. ${ }^{3-5}$ The release of protons when $\mathrm{H}_{2}$ is off is considered to occur rapidly because the heat of formation of the protonated species is much higher than that of the initial one as deduced from the MO

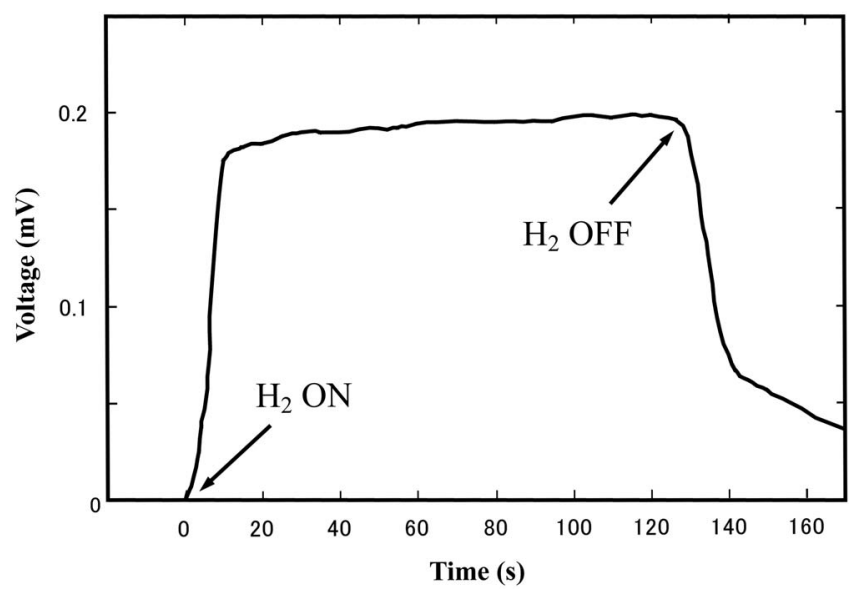

FIG. 8. Seebeck potential of the PIP-based $\mathrm{H}_{2}$ sensor when $\mathrm{H}_{2}$ is on and off. 


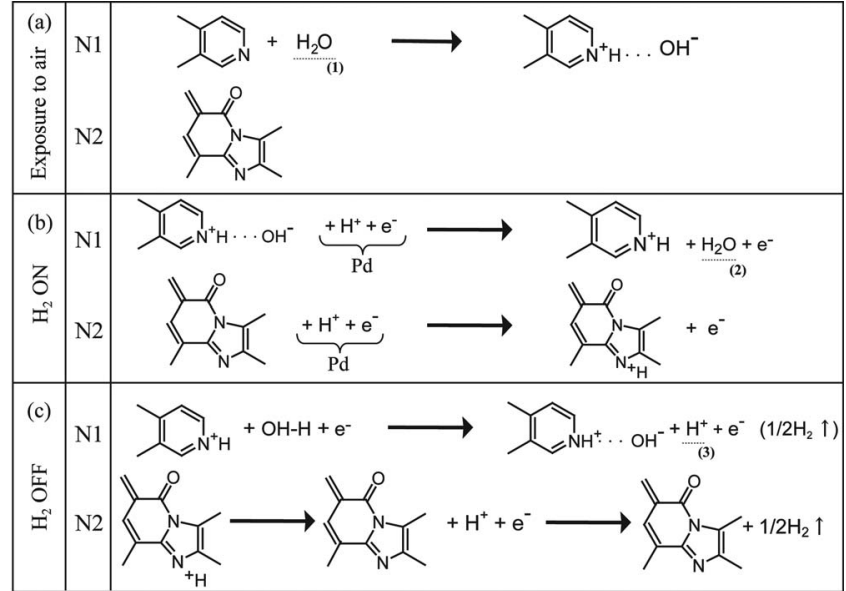

FIG. 9. Proposed mechanism for the anomalous effect of the PIP-based $\mathrm{H}_{2}$ sensor: process (a) where the color change takes place from the as prepared (blue) to the before state (violet), process (b) when $\mathrm{H}_{2}$ is on (violet $\rightarrow$ sky blue), and process (c) when $\mathrm{H}_{2}$ is shut off (sky blue $\rightarrow$ violet). (1) water moisture in air, (2) isolated water which gives noisy IR spectra in Fig. 7(a), and (3) proton current which leads to the build up of the sensor current ("anomalous effect"). $\left(\mathrm{H}^{+}+e^{-}\right)$means the dissociated proton and electron from $\mathrm{H}_{2}$ in the presence of $\mathrm{Pd}$.

calculations (Table II). On the other hand, the process occurring at the $\mathrm{N} 1$ site is assumed to be associated with the anomalous effect. In fact, this assumption is verified by the appearance of the noisy IR spectra in process (b), which is a characteristic of PIP (or PIN) as described in Sec. III D [Fig. 7(a)]. It should also be remembered that both electrons and positive carriers (presumably protons, not holes) are involved in the decay process, as shown by the Seebeck measurements. Furthermore, the longer the exposure time is, the larger and longer is the abnormal effect, as stated in sec. I. On the basis of the above results, we believe that, when $\mathrm{H}_{2}$ is shut off, the isolated water molecule reacts with $\mathrm{N}-\mathrm{H}^{+}$at the $\mathrm{N} 1$ site to give $\mathrm{NH}^{+} \cdots \mathrm{OH}^{-}$, accompanied by release of $\mathrm{H}^{+}$. The present proton contributes to the reverse of the sensor current for several hundreds of seconds (Fig. 8), as long as the protons remain unrecombined. This means that the recombination of $\mathrm{H}^{+}$with an electron proceeds rather slowly.
The anomalous effect observed in PIP- or PIN-based $\mathrm{H}_{2}$ sensors can be interpreted in terms of the protons released from the N1 site, while the decay at the N2 site is the usual process which occurs in our DPPP, ${ }^{3}$ peryleneimides (OPP, MPP, and PPP), ${ }^{4}$ and CuPPc. ${ }^{5}$

\section{CONCLUSIONS}

The anomalous phenomenon observed in PIP- or PINbased $\mathrm{H}_{2}$ sensors has been investigated on the basis of the crystal structure, molecular orbital calculations, and UVvisible/IR spectra with special attention to the sign of the charge carriers before and after $\mathrm{H}_{2}$. The conclusions drawn from the present investigation can be summarized as follows.

(1) The pyridylimidale ring integrated in PIP or PIN is responsible for the appearance of the anomalous effect.

(2) The two N sits (N1 and N2) are involved in the protonation in PIP or PIN.

(3) The N1 site is more reactive due to higher electron density than the N2. Therefore, N1 reacts immediately even with water moisture in air under normal conditions.

(4) The charge carriers solely electrons in the build up of the sensor current; whereas both electrons and protons are involved in the decay process.

(5) In the decay process, the N1 site serves to form protons with longevity which contributes to the reverse in sensor current, ending up with the appearance of the anomalous effect.

${ }^{1}$ W. Herbst and K. Hunger, Industrial Organic Pigments: Production, Properties, Applications, 3rd ed. (VCH, Weinheim, 2004).

${ }^{2}$ M. L. Perry and T. F. Fuller, J. Electrochem. Soc. 149, S67 2002.

${ }^{3}$ H. Takahashi, and J. Mizuguchi, J. Electrochem. Soc. 152, H69 (2005).

${ }^{4}$ K. Sato, K. Hino, H. Takahashi, and J. Mizuguchi, Int. J. Intell. Syst. 3, 52 (2007).

${ }^{5}$ T. Hoki, H. Takahashi, S. Suzuki, and J. Mizuguchi, IEEE Sens. J. 7, 808 (2007).

${ }^{6}$ J. Mizuguchi, Cryst. Res. Technol. 16, 695 (1981).

${ }^{7}$ K. Seeger, Semiconductor Physics, 3rd ed. (Springer-Verlag, Berlin, 1985).

${ }^{8}$ QUANTUM CACHE, version 3.2, Fujitsu, Ltd., Kanagawa, Japan.

${ }^{9}$ NIST Standard Reference Database No. 69 (National Institute of Standards and Technology, 2005). 